\title{
Sequential multimodality bronchoscopic investigation of peripheral pulmonary lesions
}

\author{
Daniel P. Steinfort ${ }^{1,2}$, Asha Bonney ${ }^{2}$, Katharine See ${ }^{2}$ and Louis B. Irving ${ }^{2}$ \\ Affiliations: \\ ${ }^{1}$ Dept of Medicine, University of Melbourne, Parkville, Australia. \\ ${ }^{2}$ Dept of Respiratory Medicine, Royal Melbourne Hospital, Parkville, Australia.
}

\section{Correspondence:}

Daniel P. Steinfort, Dept of Respiratory Medicine, Royal Melbourne Hospital, Grattan St, Parkville 3050, Victoria, Australia.

E-mail: daniel.steinfortamh.org.au

ABSTRACT Multiple guidance modalities may be combined during bronchoscopic investigation of peripheral pulmonary lesions (PPLs). The relative contribution of each modality to diagnostic performance remains uncertain.

Endobronchial ultrasound (EBUS) with virtual bronchoscopy (VB) was routinely performed, with electromagnetic navigation (EMN) utilised only where EBUS was unable to locate PPLs or where the probe was adjacent to the lesion and on-site cytologic examination was nondiagnostic.

236 consecutive patients with 245 PPLs had lesion size $22.8 \pm 12.4 \mathrm{~mm}$ (mean \pm SD). PPLs were localised using EBUS+VB alone in 188 (77\%) and was diagnostic in 134 of these (71.3\%). EBUS localisation was predicted by PPL size $(23.7 \pm 10.5$ versus $19.7 \pm 9.8 \mathrm{~mm}, \mathrm{p}=0.003)$, but not by bronchus sign, PPL-hilum distance or PPL-pleura distance. EMN in 57 patients achieved EBUS localisation in a further 17 patients (30.9\%), improving overall visualisation yield to $85 \%$. Nine of these 57 procedures achieved a definitive diagnosis (16\%), improving overall diagnostic yield to 58.4\%. Probe position and lesion type influenced overall diagnostic yield. Sensitivity for diagnosis of lung cancer was 70\% (131/188; 95\% CI 63-76\%).

Localisation rate and diagnostic sensitivity of radial probe EBUS+VB alone for diagnosis of PPLs is high. EBUS localisation rates and procedural yield are improved only modestly (by $8 \%$ and $4 \%$, respectively) with addition of EMN. Sampling following EMN should include all available methods to maximise diagnostic yield.

@ERSpublications

EMN after nondiagnostic radial EBUS gives only small incremental gain in visualisation rate and diagnostic yield http://ow.ly/SsY1U 


\section{Introduction}

Peripheral pulmonary lesions (PPLs) are a frequent presentation of early-stage lung cancer, although the probability of malignancy in such lesions can vary widely. Clinical and radiologic characteristics cannot reliably distinguish between benign and malignant nodules in most individuals [1]. Consequently, minimally invasive diagnosis is frequently performed. Diagnostic accuracy during bronchoscopic investigation is significantly improved with advanced localisation/navigation tools. Both radial probe endobronchial ultrasound (EBUS) and electromagnetic navigation (EMN) achieve high sensitivity for the detection of malignancy, with published meta-analyses reporting sensitivity of $73 \%$ and $67-71 \%$, respectively [2-4].

Bronchoscopic investigation of PPLs is associated with significantly lower rates of complications than percutaneous biopsy [5], and depending on institutional complication rates and clinical practices, cost of EBUS may be lower [6]. However, consumable costs for EMN are significant and reducing utilisation of these consumables will reduce overall healthcare costs. Isolated reports suggest combining the two techniques may achieve the highest diagnostic yields [7], although given EBUS achieves a sensitivity of $73 \%$ [2], and exceeding $84 \%$ in some reports [8-10], the cumulative gain seen following addition of EMN may not justify routine use of EMN consumables. Only one study has reported use of EMN only if peripheral lesions were unable to be localised with EBUS [11]. In that study, CheE et al. [11] described outcomes in 60 patients with a significant improvement in localisation rates, but no improvement in diagnostic yield.

We have previously reported utilising this sequential approach, as well as virtual bronchoscopy (VB), to achieve accurate placement of fiducial markers for localisation of small peripheral lung tumours [12]. Here, we describe our experience with sequential multimodality bronchoscopic investigation of PPLs. The aim of the study was to determine the incremental improvement in diagnostic performance through addition of EMN in cases where EBUS, with VB guidance, was unable to locate the lesion and to report any clinicoradiologic features that suggest EMN will improve bronchoscopic outcomes.

Methods

Approval for this study was granted by the Melbourne Health Human Research Ethics Committee. We conducted a prospective, nonrandomized observational single-centre cohort study examining consecutive patients undergoing investigation of PPLs.

\section{Performance of bronchoscopy}

Bronchoscopy was initially performed with a $4.3 \mathrm{~mm}$ diameter videobronchoscope (BF-MP160F; Olympus, Tokyo, Japan) under conscious sedation as described previously [13]. VB pre-procedure planning was performed using multiplanar formatting of Digital Imaging and Communications in Medicine (DICOM) data from computed tomography (CT) chest (slice thickness $1.0 \mathrm{~mm}$ with $0.8 \mathrm{~mm}$ overlap). Three-dimensional reconstruction of the bronchial tree from DICOM images was performed using iLogic software (SuperDimension, Plymouth, MN, USA). A bronchoscopic pathway was determined using the iLogic software and localisation of lesions was first attempted using radial EBUS as described previously, based on the "VB" pathway.

Radial EBUS examination of visible bronchial segments was based on the VB pathway. If the characteristic ultrasound signal indicating a solid lesion was identified, the EBUS probe was removed and sampling performed via the guide sheath under fluoroscopic vision. Bronchial brushings performed under fluoroscopic vision were subjected to rapid on-site cytologic evaluation (ROSE) as described previously [14]. At each location, a minimum of three brushings were performed. Where ROSE of brushings specimens did not identify malignancy, a minimum of five transbronchial lung biopsies (TBLBs) were performed. Washings were routinely performed following completion of targeted sampling with brush/forceps biopsy.

EMN bronchoscopy was performed where EBUS was unable to locate PPLs or where the probe was adjacent to the lesion and ROSE was nondiagnostic. Given probe position adjacent to the lesion is associated with reduced yield of EBUS [15-19], we reasoned that EMN may provide improved diagnostic outcomes in this scenario. In contrast, probe localisation within the target PPL is associated with a high sensitivity and therefore EMN would not further improve localisation or diagnostic performance for such lesions.

EMN was performed following insertion of a laryngeal mask airway. A larger bronchoscope (BF-P180; Olympus, Tokyo, Japan) was required to accommodate the extended working channel (EWC) within the channel of the bronchoscope. EMN was performed using the inReach system (SuperDimension, Minneapolis, MN, USA) as described previously in detail $[12,20]$. Navigation to the lesion location was undertaken, the locatable guide (SDK200; SuperDimension) was withdrawn from the EWC and repeat EBUS examination was performed. Subsequently, sampling (brushings, TBLBs, washings) was performed under fluoroscopic vision. 


\section{Determination of final diagnosis}

Diagnoses were determined where invasive sampling identified a specific histologic or cytologic diagnosis (regardless of sampling method). In patients where bronchoscopy was nondiagnostic, further investigation was directed by the primary clinician. All results of subsequent invasive investigation (e.g. percutaneous or resectional biopsy) were reviewed and recorded. Where no diagnosis was made, all patients underwent radiologic surveillance.

Benign aetiology was determined if follow-up imaging demonstrated resolution of the lesion or if stability was demonstrated for a minimum period of 12 months. Where the surveillance period was $<12$ months, the final diagnosis was recorded as indeterminate and for the purpose of determining diagnostic accuracy was identified as a false-negative procedure.

\section{Statistical analysis}

Sensitivity, specificity and accuracy of the two methods were calculated according to standard definitions. Categorical variables are presented as simple proportions and compared using Fisher's exact test. Continuous variables are expressed as mean $\pm \mathrm{SD}$, with the Mann-Whitney test used to compare unpaired nonparametric continuous values. All reported confidence intervals are two-sided. A p-value $<0.05$ was considered significant. Analyses, including multiple regression, were performed using InStat version 3.10 for Windows and QuickCalcs (GraphPad, La Jolla, CA. USA).

\section{Results}

236 consecutive patients with 245 PPLs underwent bronchoscopic investigation with EBUS+VB with or without EMN during the study period. $56 \%$ were male and mean age was 69 years. Mean lesion size was $22.8 \pm 12.4 \mathrm{~mm}$. Mean distance from lesion to hilum and lesion to pleura was $68.2 \pm 27.3$ and $21.6 \pm 15.1 \mathrm{~mm}$, respectively.

A final diagnosis of nonsmall cell lung cancer (NSCLC) was made in 188 patients (prevalence 76.7\%) and of metastatic disease in a further 14 patients (overall prevalence of malignancy 82\%). Malignant lesions had a mean diameter $23.4 \pm 10.2 \mathrm{~mm}$.

Bronchoscopy was diagnostic in 143/245 lesions (58.4\%; see table 1). Final diagnoses for patients in whom bronchoscopy was nondiagnostic $(n=102 ; 41.6 \%)$, as well as the method by which this was achieved, are recorded in table 2. Overall procedural sensitivity for diagnosis of malignancy was $67 \%(136 / 202$; $95 \%$ CI $61-74 \%)$ and sensitivity for diagnosis of lung cancer was 70\% (131/188; 95\% CI 63-76\%). Diagnostic yield for benign lesions was just $16.7 \%(7 / 42)$.

\section{Performance of EBUS+VB alone}

Localisation of PPLs was achieved using EBUS+VB alone for 188 lesions (77\% of all PPLs). The probe was positioned within the lesion in 139 of these (74\%) and adjacent to the lesion in 49 (26\%). ROSE was positive for malignancy in 114 of these (60.6\%). The procedure was diagnostic in 134 of 188 PPLs (71\%) where EMN was not utilised (table 1), therefore the yield of EBUS+VB alone was 54.7\% (134/245 PPLs).

\section{Lesion localisation with EBUS+VB alone}

Lesions localised by EBUS were significantly larger than those not localised $(23.7 \pm 10.5$ versus $19.7 \pm 9.8 \mathrm{~mm} ; \mathrm{p}=0.003)$. The localisation rate for lesions $<20 \mathrm{~mm}$ was significantly lower than for lesions $\geqslant 20 \mathrm{~mm}(70 / 103,68.0 \%$ versus $121 / 142,88.2 \%$; $\mathrm{p}=0.0025)$.

A bronchus sign could be identified in 57 PPLs (23.2\%), with 44 of these localised by EBUS (77.2\%). Localisation of lesions without a bronchus sign was not significantly different (144/148, 76.6\%; $\mathrm{p}=1.0)$. Lesion size did not differ according to the presence of a bronchus sign (23.6 \pm 10.3 with bronchus sign versus $22.6 \pm 10.6$ without; $\mathrm{p}=0.40$ ).

Lesion distance to hilum did not differ between PPLs visualised by EBUS and those not visualised $(68.75 \pm 27.5$ versus $67.09 \pm 24.08 \mathrm{~mm} ; \mathrm{p}=0.876$ ). Analysis of distance to hilum as a dichotomised variable did not identify a significantly greater likelihood of visualisation when compared at 50 and $60 \mathrm{~mm}$ ( $\mathrm{p}=0.333$ and $\mathrm{p}=0.868$, respectively).

Lesion distance to pleura did not differ between PPLs visualised by EBUS and those not visualised $(21.7 \pm 15.2$ versus $21.4 \pm 15.2 \mathrm{~mm} ; \mathrm{p}=0.962$ ). Analysis of distance to pleura as a dichotomised variable did not identify a significantly greater likelihood of visualisation when compared at 30 and $20 \mathrm{~mm}$ ( $\mathrm{p}=0.479$ and $\mathrm{p}=0.872$, respectively).

Multiple regression analysis including all above examined variables identified only lesion size as a significant influence on EBUS visualisation $(p=0.003$ ) 
TABLE 1 Diagnoses demonstrated by bronchoscopy

\begin{tabular}{lc} 
Diagnosis & $\mathbf{n}$ \\
\hline EBUS+VB alone & 188 \\
NSCLC & 126 \\
Adenocarcinoma & 77 \\
Squamous cell carcinoma & 21 \\
NSCLC NOS & 21 \\
Carcinoid & 7 \\
Metastatic disease & 5 \\
Melanoma & 2 \\
Renal cell carcinoma & 1 \\
Prostate carcinoma & 1 \\
Lacrimal gland carcinoma & 1 \\
Cryptogenic organising pneumonia & 1 \\
Sarcoidosis & 1 \\
Granulomatous inflammation & 1 \\
Nondiagnostic & 54 \\
EMN bronchoscopy & 57 \\
NSCLC & 5 \\
Adenocarcinoma & 1 \\
Adenocarcinomaף & 1 \\
Squamous cell carcinoma & 1 \\
NSCLC NOS & 2 \\
Mycobacterium avium complexף & 2 \\
Eosinophilic pneumoniaף & 1 \\
Nocardiaף & 1 \\
Nondiagnostic & 48
\end{tabular}

EBUS: endobronchial ultrasound; VB: virtual bronchoscopy; NSCLC: nonsmall cell lung cancer; NOS: not otherwise specified; EMN: electromagnetic navigation. "\#: lesion resolved on subsequent imaging; ף: diagnosis established despite not localising lesion with radial EBUS following EMN navigation.

\section{Diagnostic performance following addition of EMN}

EMN was performed in 57 patients where either EBUS was unable to locate the lesion or ROSE was nondiagnostic following identification of PPLs adjacent to a bronchus. Lesions subjected to EMN had a mean diameter $19.1 \pm 6.8 \mathrm{~mm}$ and were significantly smaller than those that underwent EBUS+VB alone $(23.8 \pm 10.7 \mathrm{~mm} ; \mathrm{p}=0.006)$. Prevalence of NSCLC in PPLs examined by EMN was lower than the overall cohort, at 63\%, and significantly lower than the cohort who underwent EBUS+VB alone (80.5\%; $\mathrm{p}=0.012)$.

EMN achieved EBUS localisation in a further 17 patients (29.8\% of patients undergoing EMN), resulting in improvement of overall visualisation yield to $85.3 \%$. Lesions visualised by EBUS following EMN were not significantly larger than those where visualisation was not achieved $(19.0+6.9$ versus $19.1+7.0 \mathrm{~mm}$; $\mathrm{p}=0.99)$. Distance from lesion to pleura and from lesion to hilum also did not differ. (21.6 \pm 12.3 versus $23.5 \pm 16.6 \mathrm{~mm} ; \mathrm{p}=0.99$ and $62.3 \pm 23.2$ versus $67.1 \pm 24.0 \mathrm{~mm} ; \mathrm{p}=0.35$, respectively).

Nine of the 57 procedures where EMN was utilised resulted in a definitive procedural diagnosis (15.8\%), improving overall diagnostic yield to $58.4 \%$. In only four EMN procedures was a diagnostic outcome clearly attributable to accurate localisation of the target lesion (as confirmed by EBUS). Five further procedures were diagnostic despite not achieving EBUS localisation (bronchial washings demonstrated adenocarcinoma on one patient, nocardia on another and Mycobacterium avuim in two, while TBLB demonstrated eosinophilic pneumonia in one). Final diagnoses of patients where EMN was utilised are presented in table 2.

Final diagnosis remained undetermined in 10/57 patients undergoing EMN (imaging < 12 months, $\mathrm{n}=7$; patient declined follow-up, $n=1$; patients lost to follow-up, $n=2$ ). Five of seven patients yet to complete 12 months of follow-up had a lesion diameter $\leqslant 19 \mathrm{~mm}$ and four of seven had stable lesion size on CT at 6 months, suggesting the true prevalence of malignancy in this cohort may be even lower than $63 \%$.

\section{Effect of lesion visualisation on diagnostic performance}

Where VB and/or EMN achieved EBUS localisation, with the probe positioned within the lesion, the procedure was diagnostic in 113/131 malignant lesions (sensitivity $86.3 \%$ ) and in $4 / 13$ benign lesions $(30.8 \%)(\mathrm{p}<0.0001)$, with overall diagnostic yield of $82.4 \%$. Where the probe was positioned adjacent to 


\begin{tabular}{|c|c|c|}
\hline Diagnosis & Method/clinical notes & $\mathbf{n}$ \\
\hline \multicolumn{2}{|l|}{ Nondiagnostic EBUS } & 54 \\
\hline \multirow[t]{2}{*}{ Benign } & Resolved on serial CT imaging & 13 \\
\hline & Stable on CT chest $>12$ months & 2 \\
\hline Non-Hodgkin's lymphoma & CT-guided biopsy & 1 \\
\hline Organising pneumonia & Surgical biopsy & 1 \\
\hline \multirow[t]{2}{*}{ Granulomatous inflammation } & CT-guided biopsy & 1 \\
\hline & Surgical biopsy & 1 \\
\hline \multirow[t]{2}{*}{ NSCLC } & CT-guided biopsy & 6 \\
\hline & Surgical biopsy & 7 \\
\hline Carcinoid & Surgical biopsy & 1 \\
\hline \multirow[t]{2}{*}{ Melanoma } & Surgical biopsy & 4 \\
\hline & CT-guided biopsy & 2 \\
\hline Prostate metastasis & CT-guided biopsy & 1 \\
\hline \multirow[t]{3}{*}{ NSCLC (presumed) } & Empiric radiation therapy & 2 \\
\hline & Serial imaging $<12$ months & 8 \\
\hline & Patient declined further investigation & 3 \\
\hline Metastatic CRC (presumed) & $\begin{array}{l}\text { New nodule in patient with prior } \\
\text { history of CRC metastases }\end{array}$ & 1 \\
\hline \multicolumn{2}{|l|}{ Nondiagnostic EBUS/EMN } & 48 \\
\hline \multirow[t]{2}{*}{ Benign } & Resolved on serial CT imaging & 12 \\
\hline & Stable on CT chest $>12$ months & 4 \\
\hline Organising pneumonia & CT-guided biopsy and subsequent resolution & 1 \\
\hline \multirow[t]{2}{*}{ NSCLC } & CT-guided biopsy & 9 \\
\hline & Surgical biopsy & 6 \\
\hline Metastatic CRC & Surgical biopsy & 1 \\
\hline \multirow[t]{4}{*}{ NSCLC (presumed) } & Empiric radiation therapy & 5 \\
\hline & Serial imaging $<12$ months & 7 \\
\hline & Patient declined further investigation & 1 \\
\hline & Lost to follow-up & 2 \\
\hline
\end{tabular}

EBUS: endobronchial ultrasound; NSCLC: nonsmall cell lung cancer; CRC; colorectal cancer; EMN: electromagnetic navigation; CT: computed tomography.

the lesion, the procedure was diagnostic in 22/46 malignant lesions (sensitivity $47.8 \%$ ) and in $1 / 9$ benign lesions (11.1\%), with overall diagnostic yield of $41.8 \%$.

Where the lesion was not visualised despite use of $\mathrm{VB}$ and/or EMN, the procedure was diagnostic in 3/29 malignant lesions (sensitivity 10.3\%) and 4/24 benign lesions (16.7\%), with overall diagnostic yield of just $9.8 \%$.

Of 202 malignant PPLs, EBUS visualisation was achieved in 175 (86.7\%), compared with 22/43 benign lesions $(51.2 \%)(\mathrm{p}<0.0001)$.

\section{Discussion}

Our findings again confirm the excellent performance of radial EBUS in evaluation of PPLs. Sensitivity for diagnosis of NSCLC was 70\%, despite a mean lesion size of only $23 \mathrm{~mm}$. Overall diagnostic yield was 58\%, which is consistent with prior studies and is lower than the observed sensitivity for NSCLC due to the poorer performance of EBUS in evaluation of benign lesions [15, 21]. We also confirm the importance of radial EBUS probe positioning, with diagnostic sensitivity and yield markedly higher where the probe was positioned within the lesion, compared with adjacent. We note a poor diagnostic performance when PPLs are unable to be visualised by the EBUS probe.

Significantly, our findings indicate that the additional value of EMN in investigation of PPLs, following failure to visualise lesions with radial EBUS, may only be very modest. EMN guidance achieved lesion visualisation in $29.8 \%$ of patients where EBUS+VB alone had not. This improved overall visualisation yield from $77 \%$ to $85 \%$. However, the diagnostic yield for lesions visualised by EBUS following EMN was just $24 \%$ and the improvement in overall procedural yield was just $4 \%$. This is consistent with the only previously published study examining performance of EMN in patients in whom radial EBUS had failed to localise the lesion. CHeE et al. [11] observed an improvement in visualisation from $75 \%$ to $93 \%$ with addition of EMN, but were only able to improve diagnostic yield by $7 \%$, consistent with our findings. 
While EMN is a navigation tool, EBUS may be considered a localisation tool with visualisation of target PPLs confirming sampling is being performed from the exact site of interest. Perhaps more importantly, EBUS allows targeting of lesions intimately related to small airways. Bronchoscopic sampling (with the exception of peripheral transbronchial needle aspiration) is confined to within the airway lumen. This may be illustrated by the observation that nonbronchogenic lesions (e.g. metastases, benign lesions), which are more likely to be parenchymally based than endoluminal, have a significantly lower yield for EBUS-directed sampling [7, 15, 19]. It is worth noting, as we have in this cohort, that even following localisation of NSCLC lesions with probe position within the target, a minority of procedures remain nondiagnostic $[15,22,23]$. This suggests that even some NSCLC lesions are not suitable for conventional endoluminal sampling techniques (brushings, forceps biopsy).

Furthermore, we observed that lesions identified (and diagnosed) by EBUS are larger and more likely to be malignant than those not localised or diagnosed by EBUS+VB. Numerous prior studies observed reducing diagnostic performance of radial probe EBUS with smaller PPL size $[2,15,18]$ and for PPLs of benign aetiology $[7,15,19]$. Presence of the bronchus sign did not correlate with diagnostic outcome in our cohort of 245 PPLs, despite this being suggested previously by other authors [22,23]. It is postulated that this sign exemplifies the intimate relationship of the PPL to the airway lumen and indicates the lesion will be amenable to bronchoscopic endoluminal sampling with forceps biopsy or cytology brush.

We suggest that EBUS is able to identify bronchoscopically amenable lesions, effectively leaving complex lesions (i.e. small, benign, no endoluminal component) for EMN to target. This would explain the poor performance of EMN among the 57 patients where EBUS+VB was unable to locate the target PPL.

Our findings strongly suggest that lesions unable to be located by radial EBUS are very unlikely to be diagnosed simply by the addition of EMN. It is worth noting that meta-analysis identifies the sensitivity of EBUS as $73 \%$ [2], whereas sensitivity for EMN alone, calculated in meta-analysis, is $67-71 \%$ [3]. This meta-analysis identified localisation with EBUS to be associated with improved yield, but did not report pooled diagnostic outcomes for studies where only electromagnetic navigational bronchoscopy was utilised.

Furthermore, there is a significant discrepancy between the rate of "successful navigation" (calculated in meta-analysis at 97\%) and diagnostic performance when EMN is utilised without EBUS [3], indicating the importance of EBUS in optimal performance of guided bronchoscopic investigation of PPLs.

In contrast to EBUS, EMN consumables are expensive, which makes their routine use impractical in most interventional pulmonology units. We therefore believe the clinically important question is whether EMN further improves upon radial EBUS, which has known high diagnostic performance, as reported by numerous authors.

We believe EMN is clearly effective in improving bronchoscopic accuracy during investigation of PPLs and the accuracy of navigation may be illustrated by the close proximity achieved through EMN-directed placement of fiducial markers [12] or pleural tattoo [24]. In cases where EBUS is unable to localise a target lesion, EMN may be particularly useful for accurate targeting of diagnostic (e.g. cryobiopsy) or therapeutic (e.g. fiducial marker placement [12] or thermal ablation [25]) interventions.

It is worth noting that in one patient, TBLB following EMN was diagnostic despite failure to successfully locate the target with EBUS. This suggests that following successful navigation, TBLB should be performed in all cases. Where EMN did not achieve EBUS localisation, we did not routinely perform TBLB. It is possible that had we done so our diagnostic performance may have been improved.

Given the proven ability of EMN to accurately navigate to the proximity of a target lesion [12], more extensive sampling techniques may significantly advance diagnostic performance of EMN, even where EBUS localisation is not achieved. Catheter aspiration in particular may be useful for lesions not visualised by EBUS $[26,27]$ and peripheral transbronchial needle aspiration may improve yield for lesions positioned adjacent to an airway [16]. We did not routinely utilise these techniques, although we recognise that doing so may have improved yield among the 55 patients who underwent EMN. The contribution of these additional sampling techniques to performance of EMN remains to be confirmed in future studies. EMN may even potentially guide more invasive forms of sampling such as bronchoscopic transparenchymal nodule access [28]. Future technical developments, such as advances in locatable guides or improvements in navigation software, may also significantly improve performance of EMN.

Our cohort comprises consecutive patients undergoing bronchoscopic investigation of PPLs, thus minimising selection bias. We had established both EBUS and EMN at our institution prior to commencement of this cohort, and therefore believe procedural proficiency was adequate to accurately reflect performance of EBUS and EMN as described. Our experience describes EMN performance for PPLs not amenable to radial EBUS and we note the concordance of our findings with the sole prior study to examine EMN performance in this specific patient cohort. 
Our results are not reflective of the performance of EMN when utilised initially in bronchoscopic investigation of PPLs. Previous reports suggest good diagnostic performance of EMN without EBUS [3]. At centres where EBUS is not available, EMN-guided bronchoscopy should remain the preferred first investigation for PPLs, given its good diagnostic performance and the favourable safety performance of bronchoscopic sampling compared with percutaneous methods [5].

We used multiple tools to optimise our diagnostic outcomes for radial EBUS. Our centre routinely utilises fluoroscopy and ROSE in performance of radial EBUS. We have previously published that ROSE may improve diagnostic yield of radial EBUS [14], while most published studies on radial EBUS report use of fluoroscopic guidance, consistent with our belief in its value to optimise performance of radial EBUS [29]. Furthermore, multiple studies have demonstrated that VB may improve diagnostic performance of radial EBUS $[10,30,31]$. In centres where these adjunct tools are not utilised, the incremental gain in diagnostic performance with addition of EMN may be greater than observed in our current study.

Previous studies have noted the highest yield for bronchoscopy is seen where EBUS and EMN are combined $[7,11,27]$. This is our conclusion also, although the contribution of EMN in addition to EBUS appears modest for assessment of the difficult lesions EBUS is unable to identify. Given the cost of EMN consumables, and our observed low yield of EMN for such lesions, future studies are required to confirm the clinicoradiologic characteristics that predict diagnostic outcomes for EMN following negative EBUS and to confirm the best sequence of bronchoscopic modalities in such cases.

\section{Conclusions}

Localisation rate and diagnostic sensitivity of radial probe EBUS+VB alone for diagnosis of PPLs are high. Lesions not localised by EBUS are smaller and more likely to be benign that those visualised by EBUS, which likely contributes to the significantly lower diagnostic yield of EMN for lesions not located by EBUS, although overall EBUS localisation rates and diagnostic yield are improved modestly (by $8 \%$ and 4\%, respectively) with addition of EMN. Sampling following EMN should include all available methods, including standard TBLB, to maximise diagnostic yield. Following nondiagnostic EBUS, alternative nonbronchoscopic techniques for sampling should be considered. Improved sampling tools and techniques are required to advance diagnostic performance of bronchoscopy.

\section{References}

1 Gould MK, Donington J, Lynch WR, et al. Evaluation of individuals with pulmonary nodules: when is it lung cancer? Diagnosis and management of lung cancer, 3rd ed: American College of Chest Physicians evidence-based clinical practice guidelines. Chest 2013; 143: 5 Suppl., e93S-e120S.

2 Steinfort DP, Khor YH, Manser RL, et al. Radial probe endobronchial ultrasound for the diagnosis of peripheral lung cancer: systematic review and meta-analysis. Eur Respir J 2011; 37: 902-910.

3 Gex G, Pralong JA, Combescure C, et al. Diagnostic yield and safety of electromagnetic navigation bronchoscopy for lung nodules: a systematic review and meta-analysis. Respiration 2014; 87: 165-176.

4 Wang Memoli JS, Nietert PJ, Silvestri GA. Meta-analysis of guided bronchoscopy for the evaluation of the pulmonary nodule. Chest 2012; 142: 385-393.

5 Steinfort DP, Vincent J, Heinze S, et al. Comparative effectiveness of radial probe endobronchial ultrasound versus CT-guided needle biopsy for evaluation of peripheral pulmonary lesions: a randomized pragmatic trial. Respir Med 2011; 105: 1704-1711.

6 Steinfort DP, Liew D, Irving LB. Radial probe EBUS versus CT-guided needle biopsy for evaluation of peripheral pulmonary lesions: an economic analysis. Eur Respir J 2013; 41: 539-547.

7 Eberhardt R, Anantham D, Ernst A, et al. Multimodality bronchoscopic diagnosis of peripheral lung lesions: a randomized controlled trial. Am J Respir Crit Care Med 2007; 176: 36-41.

8 Asano F, Matsuno Y, Tsuzuku A, et al. Diagnosis of peripheral pulmonary lesions using a bronchoscope insertion guidance system combined with endobronchial ultrasonography with a guide sheath. Lung Cancer 2008; 60: 366-373.

9 Yoshikawa M, Sukoh N, Yamazaki K, et al. Diagnostic value of endobronchial ultrasonography with a guide sheath for peripheral pulmonary lesions without X-ray fluoroscopy. Chest 2007; 131: 1788-1793.

10 Asano F, Shinagawa N, Ishida T, et al. Virtual bronchoscopic navigation improves the diagnostic yield of radial-endobronchial ultrasound for peripheral pulmonary lesions with involved bronchi on CT. Intern Med 2015; 54: 1021-1025.

11 Chee A, Stather DR, Maceachern P, et al. Diagnostic utility of peripheral endobronchial ultrasound with electromagnetic navigation bronchoscopy in peripheral lung nodules. Respirology 2013; 18: 784-789.

12 Steinfort DP, Siva S, Kron T, et al. Multimodality guidance for accurate bronchoscopic insertion of fiducial markers. J Thorac Oncol 2015; 10: 324-330.

13 Steinfort DP, Irving LB. Patient satisfaction during endobronchial ultrasound-guided transbronchial needle aspiration performed under conscious sedation. Respir Care 2010; 55: 702-706.

14 Steinfort DP, Leong TL, Laska IF, et al. Diagnostic utility and accuracy of rapid on-site evaluation of bronchoscopic brushings. Eur Respir J 2015; 45: 1653-1660.

15 Tay JH, Irving L, Antippa P, et al. Radial probe endobronchial ultrasound: factors influencing visualization yield of peripheral pulmonary lesions. Respirology 2013; 18: 185-190.

16 Chao TY, Chien MT, Lie CH, et al. Endobronchial ultrasonography-guided transbronchial needle aspiration increases the diagnostic yield of peripheral pulmonary lesions: a randomized trial. Chest 2009; 136: 229-236. 
Yamada N, Yamazaki K, Kurimoto N, et al. Factors related to diagnostic yield of transbronchial biopsy using endobronchial ultrasonography with a guide sheath in small peripheral pulmonary lesions. Chest 2007; 132: 603-608.

18 Chen A, Chenna P, Loiselle A, et al. Radial probe endobronchial ultrasound for peripheral pulmonary lesions. A 5-year institutional experience. Ann Am Thorac Soc 2014; 11: 578-582.

19 Chung $\mathrm{YH}$, Lie $\mathrm{CH}$, Chao TY, et al. Endobronchial ultrasonography with distance for peripheral pulmonary lesions. Respir Med 2007; 101: 738-745.

20 Leong S, Ju H, Marshall H, et al. Electromagnetic navigation bronchoscopy: a descriptive analysis. J Thorac Dis 2012; 4: 173-185.

21 Oki M, Saka H, Kitagawa C, et al. Endobronchial ultrasound-guided transbronchial biopsy using novel thin bronchoscope for diagnosis of peripheral pulmonary lesions. J Thorac Oncol 2009; 4: 1274-1277.

22 Guvenc C, Yserbyt J, Testelmans D, et al. Computed tomography characteristics predictive for radial EBUSminiprobe-guided diagnosis of pulmonary lesions. J Thorac Oncol 2015; 10: 472-478.

23 Evison M, Crosbie PA, Morris J, et al. Can computed tomography characteristics predict outcomes in patients undergoing radial endobronchial ultrasound-guided biopsy of peripheral lung lesions? J Thorac Oncol 2014; 9: 1393-1397.

24 Wallbridge $\mathrm{P}$, Tay A, Russel P, et al. Electromagnetic navigation bronchoscopy directed pleural tattoo to aid surgical resection of peripheral pulmonary lesions. J Bronchology Interv Pulmonol 2015; in press [DOI: 10.1097/ LBR.0000000000000214].

25 Narsule CK, Sales Dos Santos R, Gupta A, et al. The efficacy of electromagnetic navigation to assist with computed tomography-guided percutaneous thermal ablation of lung tumors. Innovations 2012; 7: 187-190.

26 Peschke A, Wiedemann B, Hoffken G, et al. Forceps biopsy and suction catheter for sampling in pulmonary nodules and infiltrates. Eur Respir J 2012; 39: 1432-1436.

27 Eberhardt R, Morgan RK, Ernst A, et al. Comparison of suction catheter versus forceps biopsy for sampling of solitary pulmonary nodules guided by electromagnetic navigational bronchoscopy. Respiration 2010; 79: 54-60.

28 Herth FJ, Eberhardt R, Sterman D, et al. Bronchoscopic transparenchymal nodule access (BTPNA): first in human trial of a novel procedure for sampling solitary pulmonary nodules. Thorax 2015; 70: 326-332.

29 Steinfort D, Irving L. Use of fluoroscopy during endobronchial ultrasonography for transbronchial lung biopsies of peripheral lung lesions. J Thorac Oncol 2008; 3: 685-686.

30 Ishida T, Asano F, Yamazaki K, et al. Virtual bronchoscopic navigation combined with endobronchial ultrasound to diagnose small peripheral pulmonary lesions: a randomised trial. Thorax 2011; 66: 1072-1077.

31 Wong KY, Tse HN, Pak KK, et al. Integrated use of virtual bronchoscopy and endobronchial ultrasonography on the diagnosis of peripheral lung lesions. J Bronchology Interv Pulmonol 2014; 21: 14-20. 